\title{
Breakdown Phenomena in Water Vapor Microdischarges
}

\author{
M. Radmilović-Radjenović, B. Radjenović* And M. Savić \\ Institute of Physics, Pregrevica 118, 11080 Belgrade, Serbia
}

\begin{abstract}
The gas breakdown at the large gap sizes is reasonably well understood. However, the breakdown phenomenon in microgaps is still not sufficiently explored. The high electric fields realized in small gaps combined with the lowering of the potential barrier, seen by the electrons in the cathode as ion approaches lead to ion-enhanced field emission leading to deviations from the standard Paschen law. In this paper, semi-empirical expressions for the breakdown voltage based on the fitting of numerical solutions of the DC breakdown criteria in microdsicharges have been derived. In the standard breakdown criteria the secondary emission coefficient that incorporates the enhancement of the secondary electron emission has been included. The obtained expressions can be used for determination the pressure and the gap dependence of the breakdown field strength in the water vapor, separately.
\end{abstract}

PACS numbers: 52.50.Qt, 52.65.Rr, 52.80.Pi

\section{Introduction}

In the past few decades the field of microdischarges have become more common in everyday life. Microplasmas ecompase the adventages of low-pressure plasmas with the advenatges of being micro [1-4]. Due to their portability and the non-equilibrium character of the discharges, microplasmas are finding application in many research disciplines, from the optimizing the plasma screens [5], localized silicon etching [6], tunable UV source [7], gas spectroscopy [8,9], spectroscopy of water impurities [10], up to localized treatment of materials and assembly of nanostructures [11]. On the other hand, plasma-based microsystems can find application in bio microelectromechanical system (bio-MEMS) sterilization, small-scale materials processing and microchemical analysis systems [12]. However, integrability requires not only a reduction in size, but also an understanding of the physics governing the new small-scale discharges.

Making the gap small is the easiest way to obtain a big force, which is restricted by the electric field of breakdown $[13,14]$. It is necessary to be aware of the breakdown voltage in microgaps. When changing the size of plasmas, there are scaling laws that are helpful in determining the operating parameters of various sizes of plasmas. The fact that microdischarges operate under conditions, where boundary effects dominate, indicates the importance of establishing scaling laws in a such small gaps. The best way is perhaps to start from the low pressure discharges and to employ the standard scaling laws [15]. The motivation for our studies, resulting from the fact that the electrical breakdown in microgaps occurs at voltages far below the pure Paschen curve mini-

* corresponding author; e-mail: bradjeno@ipb.ac.rs mum and that the modified Paschen curve should be used instead for micron and sub-micron gaps [16]. Electrons from the field emission are one of the possible reasons why the breakdown and sparks occur in a vacuum, which of course is not possible if one only considers the Townsend avalanche mechanisms for the gas phase and the surface ionization that are normally used to generate the Paschen curve [17].

Microplasma can be generated in a wide range of the pressure. In the large scale systems, the experimentally observed Paschen law has been successfully explained by the Townsend theory [18]. The Paschen's law is based on the observation that, over a large range of the pressures and electrode separations, the probability of the ionization per collision in the gas and the probability of the production of electrons by ions by a secondary process are both dependent on the average kinetic energy of the electrons and ions and therefore on the reduced electric field $E / N$ (the electric field $\mathrm{E}$ to the gas number ratio $N$ ) [19]. The Townsend mechanism by which successive ionizations of the gas molecules induce the gas breakdown describes the process satisfactorily at large separations [20]. The significant parameter is $p d$ (the product of the gap distanced and the pressure $P$ ). Typically, the Townsend's mechanism (and by extension Paschen's law) applies at $p d$ products less than 1000 Torr $\mathrm{cm}$, or gaps around a $1 \mathrm{~cm}$ at $1 \mathrm{~atm}$ [21].

The mechanism of the electrical breakdown is, however, completely different in microgaps $[2,3,4,14,15]$. A rapid fall of the breakdown voltage with decreasing the gap size may be attributed to the onset of the ion-enhanced field emission in microgaps. Violations of the similarity law take place for the left hand branch of the curve, for such $p d$ values where the electron mean free path is comparable with the gap. When the electron mean free path is comparable with the electrode sepa- 
ration the electrical breakdown is based on the cathode-induced breakdown model.

Water vapour in the atmosphere is the key trace gas controlling weather and climate. It also plays a central role in atmospheric chemistry, influencing the heterogeneous chemical reactions that destroy stratospheric ozone. The effects of water vapour are large in the upper troposphere and lower stratosphere, but there are few measurements of water vapour concentrations and its long-term variation in this altitude only region. The influence of water vapour on the breakdown voltage of uniform field gaps has been investigated by Ritz1 [22], who was found that the breakdown voltage in air increases by $2 \%$ for a change in the partial pressure of water vapour. Letter on, some results indicated that the presence of water vapour raised the breakdown voltage by $2.7 \%$ above that of dry air [23]. Allen and Phillips [24] investigated the effect of humidity on the spark breakdown voltage. Recently, measurements have been performed for low-pressure breakdown in water vapor [25].

In this paper, high-pressure breakdown field strength in water vapor has been theoretically studied [26]. The expressions for the DC breakdown criteria including the ion-enhanced secondary emission coefficient has been numerically solved for water vapor in order to determine the breakdown voltage versus the gap spacing and the pressure, respectively. The results of this research can be applied in the construction of compact pulse power generators for bioelectric applications.

\section{Semi-empirical expressions}

Combining the expressions for the electron yield [13]:

$$
\gamma=K \mathrm{e}^{-D / E} \text {, }
$$

and for the ionization coefficient [27]:

$$
\alpha / p=A \mathrm{e}^{-B p / E},
$$

with the expression for the DC breakdown criteria:

$$
\gamma\left(\mathrm{e}^{\alpha d}-1\right)=1 \text {, }
$$

we obtain the transcendental equation [28]:

$$
K \mathrm{e}^{-D / E}\left(\mathrm{e}^{A p d \mathrm{e}^{-B p / E}}-1\right)=1,
$$

where the material dependent constant $D$ can be calculated using the expression:

$$
D=6.85 \times 10^{7} \phi^{3 / 2} / \beta,
$$

knowing a field enhancement factor $\beta$ and the work function of the metal $\phi$ expressed in $\mathrm{eV}$. On the other hand, from the experimental values of the slope of the $\log \gamma$ versus $1 / E$, the values of $D$ were also determined for some materials, for example, aluminum, stainless steel and conventional steel (as can be seen, from Table I in Ref. [13]). In this paper we have used the value of $9.3 \times 10^{8} \mathrm{~V} / \mathrm{m}$ that corresponds to the aluminum. Contrary, determination of the constant $K$ that appears in Eqs. (1) and (4) may be quite difficult, especially if there are additional complications due to electron attachment, ionization by metastables, ect. [14]. In practice, the constant $K$ can be found from the ratio of the field emis- sion current density to the positive ion current density onto the cathode. $A$ and $B$ are gas dependent constants that can be found elsewhere [27]. The expressions for the breakdown field strength will be determined by solving transcendental Eq. (4) numerically by using the package Mathematica and then by fitting these solutions.

\section{Results and discussion}

For a fixed pressure, the transcendental Eq. (4) has been numerically solved by varying the interelectrode distance $d$. The obtained numerical solutions for the breakdown field strength $E$ against the gap size $d$ are shown in Fig. 1. These values can be fitted by a simple formula:

$$
E=a d^{-1}+b \times d^{c},
$$

where the fitting coefficients $a, b$ and $c$, for the a few pressures are listed in Table I.

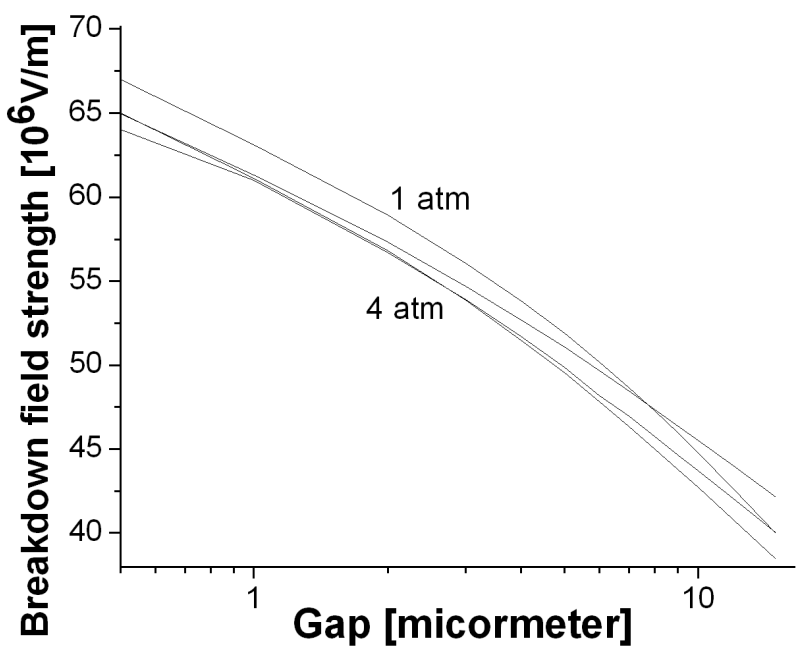

Fig. 1. The breakdown field strength versus the gap spacing for pressure in the range from $1 \mathrm{~atm}$ up to $4 \mathrm{~atm}$. The gap was varied between $0.5 \mu \mathrm{m}$ up to $15 \mu \mathrm{m}$.

TABLE I

Fitting coefficients for the Eq. (6) for various values of the pressure.

\begin{tabular}{c|c|c|c}
\hline \hline Pressure $[\mathrm{atm}]$ & $a$ & $b$ & $c$ \\
\hline 1 & -26.122 & 90.012 & -0.281 \\
2 & -20.662 & 82.597 & -0.267 \\
3 & -14.906 & 76.304 & -0.228 \\
4 & -11.456 & 73.16 & -0.196
\end{tabular}

As can be seen from Fig. 1, the breakdown field strength defined as the ration of the breakdown voltage and the gap size strongly depends on the interelectrode separation. For gaps less than $5 \mu \mathrm{m}$, the breakdown phenomena are attributed to the ion-enhanced field emission [14]. At large separations, however, processes in 
TABLE II

The Values of the fitting coefficients $m$ and $n$, for a few gap sizes, in Eq. (7).

\begin{tabular}{c|c|c}
\hline \hline Gap $[\mu \mathrm{m}]$ & $m$ & $n$ \\
\hline 0.5 & 90.188 & -0.0442 \\
1 & 85.898 & -0.0456 \\
2 & 83.266 & -0.052 \\
4 & 82.527 & 0.0574
\end{tabular}

the gas cause breakdown rather then secondary electron emission.

In a similar way, for a fixed gap sizes, the transcendental Eq. (4) has also been solved by varying the gas pressure, in order to obtain the pressure dependence of the electric field strength. Numerical solutions are presented in Fig. 2 and fitted by using expression:

$$
E=m \cdot p^{n},
$$

with fitting coefficient $m$ and $n$ given in Table II.

Figure 2 clearly illustrates the weak dependence of the breakdown field strength on the pressure, even for the gaps of the order of a few micrometers. Such trend in the curves presented in Fig. 2 can be explained by the exponential dependence of the ion-enhanced field emission on the electric field and not on the pressure.

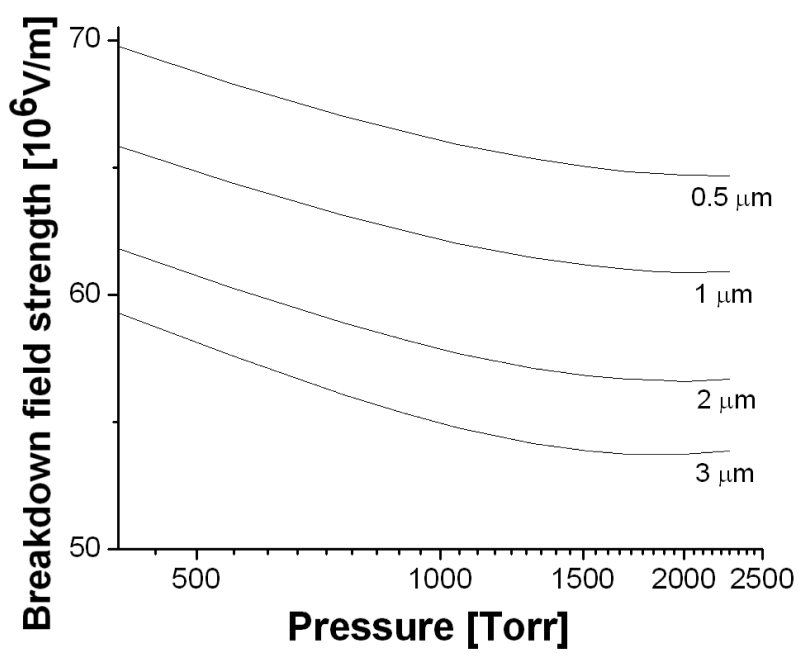

Fig. 2. The breakdown field strength as a function of the gas pressure for the gaps from 0.5 to $3 \mu \mathrm{m}$.

\section{Conclusion}

This paper contains theoretical studies of the breakdown field strength in the microgaps. Departures from the large scale similarity laws are expected with the onset of field emission on such small gaps indicating that Townsend mechanism is not sufficient to explain the breakdown mechanism. The semi-empirical expressions for the breakdown filed strength suggested here are based on the fit of the numerical solutions of the breakdown criteria including field emission effects in microgaps. The new breakdown field curve obtained, when the field emissions are accounted for, retains the right branch of the conventional Paschen curve, i.e. field emissions can be neglected in large gaps. The left branch, however, is substituted by a rapid decrease of the breakdown voltage and increases in the breakdown field strength below the minimum of the conventional Paschen curve. This reduction in the breakdown voltage is observed for gap sizes smaller than $5 \mu \mathrm{m}$ and is a direct consequence of the onset of field emissions.

The results of our studies should be useful for determining minimum ignition voltages in microplasma sources as well as the maximum safe operating voltage and critical dimensions in other microdevices.

\section{Acknowledgments}

The authors acknowledge financial support of the Ministry of Science of the Republic of Serbia 141025.

\section{References}

[1] J.M. Thores, R.S. Dhariwal, Nanotechnology 10, 102 (1999).

[2] M. Radmilović-Radjenović, J.K. Lee, F. Iza, G.Y. Park, J. Phys. D, Appl. Phys. 38, 950 (2005).

[3] M. Radmilović-Radjenović, B. Radjenović, Contrib. Plasma Phys. 47, 165 (2007).

[4] M. Radmilović-Radjenović, B. Radjenović, Plasma Sources Sci. Technol. 16, 337 (2007).

[5] S.S. Yang, J.K. Lee, S.W. Ko, H.C. Kim, J.W. Shon, Contrib. Plasma Phys. 44, 536 (2004).

[6] H. Wang, G. Li, L. Jia, L. Li, G. Wang, Chem. Commun. (Camb) 7, 3786 (2009).

[7] P. Kurunczi, J. Lopez, H. Shah, K. Becker, International Journal of Mass Spectroscopy 205, 277 (2001).

[8] T. Svensson, M. Andersson, L. Rippe, J. Johansson, S. Folestad, S. Andersson-Engels, Opt. Lett. 33, 80 (2008).

[9] M. Saito, T. Hiraga, M. Hattori, S. Murakami, T. Nakai, Magn. Reson. Imaging 23, 607 (2005).

[10] L. Que, C.G. Wilson, Y.B. Gianchandani, J. Microelectromech. Syst. 14, 185 (2005).

[11] A.K. Chakraborty, A.J. Golumbfskie, Annual Review of Physical Chemistry 52, 537 (2001).

[12] W.K.T. Coltro, Quimica Nova 30, 1986 (2007).

[13] W.S. Boyle, P. Kisliuk, Phys. Rev. 97, 255 (1955).

[14] M. Radmilović-Radjenović, B. Radjenović, Plasma Sources Sci. Technol. 17, 024005 (2008).

[15] Z.Lj. Petrović, N. Škoro, D. Marić, C.M.O. Mahony, P.D. Maguire, M. Radmilović-Rađenović, G. Malović, J. Phys. D, Appl. Phys. 41, 194002 (2008).

[16] L.H. Germer, J. Appl. Phys. 30, 46 (1959).

[17] P. Kisliuk, J. Appl. Phys. 30, 51 (1959).

[18] F. Paschen, Wied. Ann. 37, 69 (1889). 
[19] L.B. Loeb, Fundamental Processes of Electrical Dishcarges in Gases, J. Wiley and Sons, Inc., New York (1939).

[20] J.M. Meek, J.D. Craggs, Electrical breakdown of gases, Oxford Unversity Press, (1953).

[21] M.A. Lieberman, A.J. Lichtenberg, Principles of Plasma Discharges and Materials Processing 2nd ed. Wiley Interscience, Hoboken, NJ: Wiley, (2005).

[22] H. Ritz, Archiv fur Elektrotechnik 26, 219 (1932).

[23] W. Kohrmann, Ann. d. Phys. 18, 379 (1956).

[24] K.R. Allen, K. Phillips, Nature 183, 174 (1959).
[25] D. Marić, N. Škoro, G. Malović, Z.Lj. Petrović, Proceedings of the 29th ICPIG, Cancun Mexico, (12-17 July 2009), PA8-5 (2009).

[26] M. Radmilović-Radjenović, B. Radjenović, Contributed papers of 24th Summer School and International Symposium on the Physics of Ionized Gases, Novi Sad, Serbia (25-29 August 2008) 379 (2008).

[27] D. Marić, M. Radmilović-Radjenović, Z.Lj. Petrović, EPJ D 35, 313 (2005).

[28] M. Radmilović-Radjenović, B. Radjenović, Europhys. Lett. 83, 25001 (2008). 